\title{
A Study of Milk Particles Size Variation with pH Change using Dynamic Light Scattering
}

\author{
Dan Chicea $^{{ }^{*}}$, and Liana Maria Chicea $^{2}$ \\ ${ }^{1}$ Research Center for the Physics of Complex Systems, Faculty of Sciences, Lucian Blaga University \\ of Sibiu, Dr. Ion Ratiu str., no. 5-7, Sibiu, 550012, România \\ ${ }^{2}$ Faculty of Medicine, Lucian Blaga University of Sibiu, Dr. Ion Ratiu str., no. 5-7, Sibiu, 550012, \\ România
}

\begin{abstract}
A very simple experimental setup for a Dynamic Light Scattering measurement was used to measure the average size of the milk proteins in aqueous suspensions at $20^{\circ} \mathrm{C}$. The $\mathrm{PH}$ of the suspensions was adjusted using Calcium lactate, in its most common form of pentahydrate $\mathrm{C} 6 \mathrm{H} 10 \mathrm{CaO} 6 \cdot 5 \mathrm{H} 2 \mathrm{O}$. The mean size variation of the suspended particles in time has been monitored and reveals a fast increase over a time interval of less than ten of seconds.
\end{abstract}

\section{Introduction}

Bovine milk (milk hereafter), contains fat globules with a diameter of about $2 \mu \mathrm{m}$, casein micelles of about $0.2 \mu \mathrm{m}$, whey proteins of $0.006 \mu \mathrm{m}$, and many other small molecules having the diameter less than $0.002 \mu \mathrm{m}$ [1]. Milk proteins constitute approximately $3.0 \%-$ $3.5 \%$ of bovine milk. About $80 \%$ of bovine milk protein is called casein, while the rest are named whey protein [2].

The medical drug industry and traditional industrial food industry use the self-assembly processes under different physical conditions, as temperature and pressure and chemical conditions as $\mathrm{pH}$. For this reason, studying the dynamics of the milk protein size influence of the $\mathrm{pH}$ might be of interest. As the aggregation or denaturation can undergo quite fast, it is of interest to use an optical method, especially one that can be used in situ, without removing the sample from the protein aggregation reaction vessel and without using reagents that can modify the $\mathrm{pH}$ of the solution.

When a light beam is incident on a fluid containing suspended particles, each particle scatters light and therefore becomes a secondary light. The particles act like scattering centers (SC). If the incident light beam is coherent, the scattered waves will interfere in the far field. The consequence of the complex motion of the SCs is the dynamic character of the far interference field, which appears as "boiling speckles". Several articles report on the variation of several parameters, as the average intensity, speckle size and speckle contrast, with the size and the concentration of the SCs, references [3 - 5] being some of them, but they are not optimal for particle sizing in dynamic processes where both the number of SCs

* Corresponding author: dan.chicea@ulbsibiu.ro 
and the SCs diameter can change in time [5]. The physical method that uses the dependence between the speckle dynamics and the Brownian motion is called Photon Correlation Spectroscopy (PCS) or Dynamic Light Scattering (DLS) and the theoretical basis of the method is explained in many works, [6 - 10] being just some of them. The next sections present the procedure used in preparing the milk suspension, a brief description of the DLS experimental setup and of the DLS time series processing to obtain the average aggregates diameter. Another section will present the results and a discussion on them, followed by a concluding section.

\section{Materials and methods}

\subsection{Sample preparation}

Bovine skimmed milk, $1.5 \%$ fat was used. In order to perform a light scattering experiment, the suspension has to be transparent, therefore using milk as it is will not enable recording a time series, as it will be detailed in the next subsection.

For each sample, $0.05 \mathrm{ml}$ of milk were diluted in $3.45 \mathrm{ml}$ of deionized water, introduced in a circular glass tube and placed in the DLS sample holder.

In order to modify the $\mathrm{pH}$ of the suspension $0.4620 \mathrm{~g}$ of Calcium lactate, in its most common form of pentahydrate, $\mathrm{C} 6 \mathrm{H} 10 \mathrm{CaO} 6 \cdot 5 \mathrm{H} 2 \mathrm{O}$, were dissolved in $40 \mathrm{ml}$ of deionized water. A quantity of $0.5 \mathrm{ml}$ of Calcium lactate solution was aspired into a $1 \mathrm{ml}$ syringe. DLS time series recording was initiated and after the first time series recording was completed, the solution was injected fast into the glass tube containing the milk suspension. The $\mathrm{pH}$ of this sample, measured after the DLS experiment was completed, was 7.0.

\subsection{DLS procedure}

The DLS technique has been developed for a long time [6 - 10], and is quite well established, therefore the theory behind it is not repeated here, but the experimental setup and the main steps in assessing the average particle size using DLS are presented briefly in the following paragraphs.

The experimental setup is presented in Fig. 1 and consists of a laser source, which was a Laser diode, $15 \mathrm{~mW}$, with the wavelength of $633 \mathrm{~nm}$, working in continuous regime, a circular glass tube for the liquid sample, a detector, a preamplifier with a linear response in the audio frequency range $20-20000 \mathrm{~Hz}$, a PC for recording and later on processing the time series.

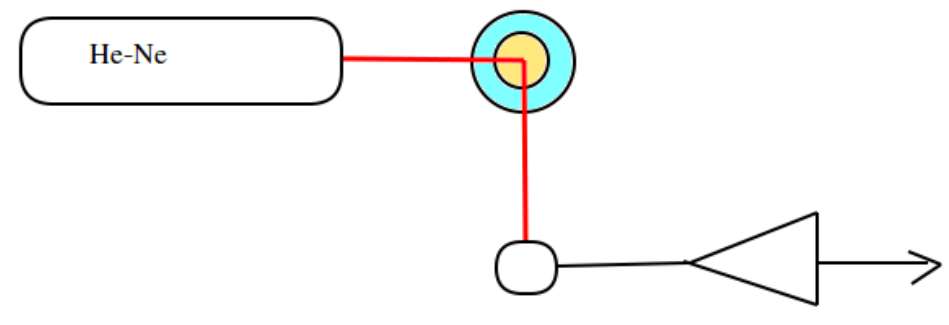

Detector Preamplifier To PC

Fig. 1. The DLS experimental setup 
The scattering angle was chosen to be $90^{\circ}$, which is typical for DLS experiments [6], [7], [9], [11-13]. The data acquisition rate was $16 \mathrm{KHz}$, which was sufficient for the particles range covered in this work.

The data acquisition system (DAS) produces a sequence of values recorded at equal time intervals $\Delta \mathrm{t}$, as in Eq. (1), succession called a time series:

$$
I(0), I(\Delta \mathrm{t}), \mathrm{I}(2 \Delta \mathrm{t}), \ldots, \mathrm{I}(\mathrm{n} \Delta \mathrm{t}), \ldots
$$

If the suspended particles are considered to be monodispersed, the autocorrelation of the time series (ACR hereafter) has a simplified form [6], [11-13], [14]:

$$
G(\tau)=\mathrm{Ae}^{-2 \mathrm{Dq}^{2} \tau}+\mathrm{B}
$$

Where:

$$
\mathrm{q}=\frac{4 \pi \mathrm{n}}{\lambda} \sin \left(\frac{\theta}{2}\right)
$$

In (3) $n$ is the refractive index of the carrier fluid, $\lambda$ is the wavelength of the laser incident light and $\theta$ is the scattering angle, which was chosen to be $90^{\circ}$ for this work, typical for DLS measurements in liquid suspensions.

The ACR can be further on normalized in two small steps. Firstly, constant B is subtracted, which makes ACR to be zero-based. Secondly, an experimental adjustment is made in such a manner that the detector will cover a single speckle, as described in detail in [11-13] and [14], making the spatial coherence factor, included in A for this particular case, to be equal to 1 . After normalization, the ACR has a simple form depending on a single parameter, which is $\mathrm{D}$ :

$$
G(\tau)=\mathrm{e}^{-2 \mathrm{Dq}^{2} \tau}
$$

The diffusion coefficient $\mathrm{D}$ is related to Boltzmann`s constant, $\mathrm{k}_{\mathrm{B}}$, which is related to $\eta$, the dynamic viscosity coefficient of the solvent, $T$ the absolute temperature of the sample and $\mathrm{d}$ the hydrodynamic diameter of the particle, as described by Einstein-Stokes relation [15]:

$$
\mathrm{D}=\frac{k_{B} T}{3 \pi \eta \mathrm{d}}
$$

A plot of the ACR of a time series is illustrated in Fig. 2.

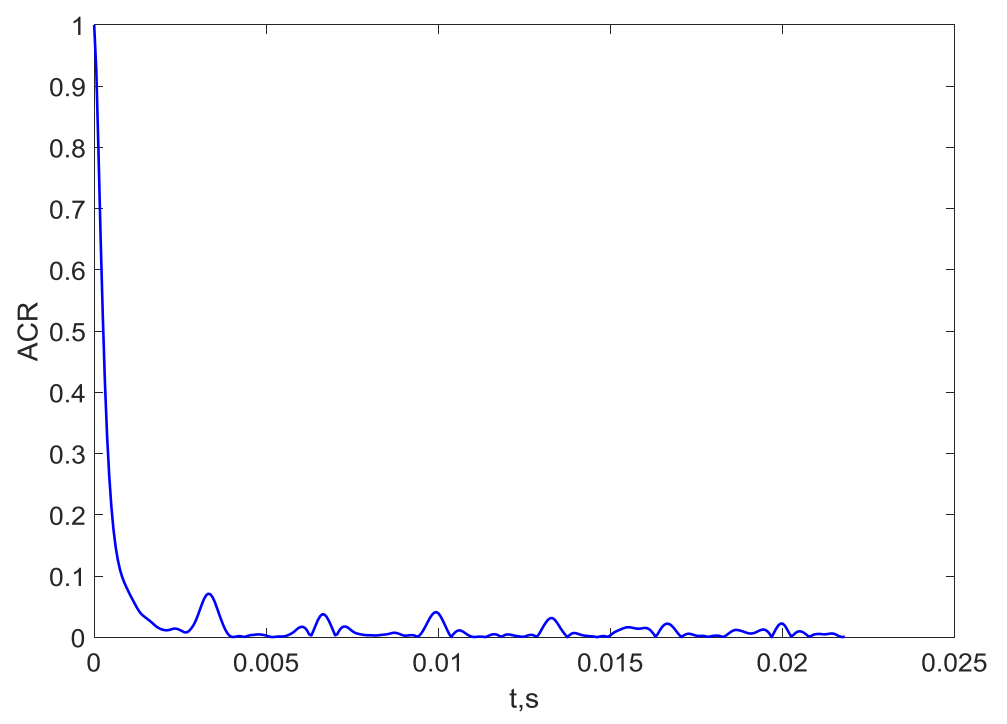

Fig. 2. The ACR of a time series 
Examining figure 2 we notice that the ACR exhibits a fast decrease with several small peaks, which are produced by the noise in the time series, as it is not filtered. The ACR does not exhibit plateaus, therefore the assumption of having relatively mono-dispersed particles in suspension is correct.

A Matlab code was written and used to record the DLS time series [11-13]. The time length of the time series and the delay between consecutive recordings are adjustable parameters. Several sets of time series were recorded with a small delay and some with a bigger delay. Each time series was processed by computing the normalized ACR and by fitting a function described by Eq. (4) with D substituted from (5) in it to the ACR, using a nonlinear least squares minimization procedure. The output was the average diameter $\mathrm{d}$ for each time series.

\section{Results and discussions}

Following the preparation steps described in subsection 2.1, the samples were placed in the circular glass tube after dilution. The DLS time series recording was initiated and the solution was injected fast right after the first recording was completed. The average diameters for each time series and are plotted versus time $t$, elapsed from the beginning of the first recording presented in Fig. 3. The $\mathrm{pH}$ of the solution was 7.

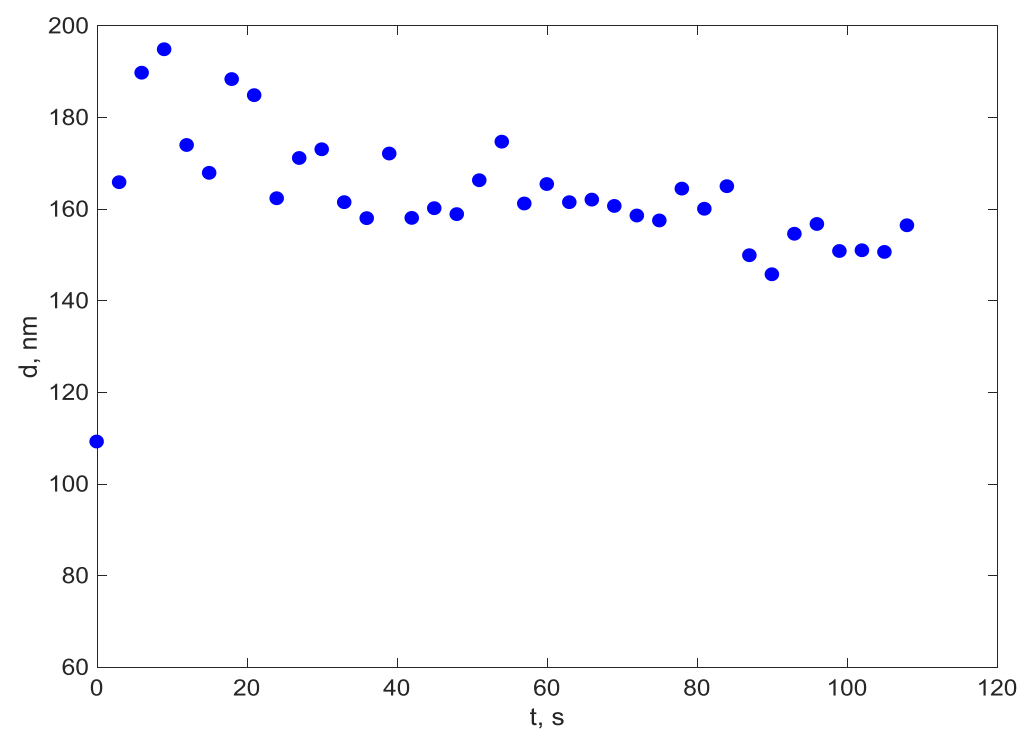

Fig. 3. The variation of the average protein aggregates diameters in time

The $\mathrm{pH}$ of bovine milk is typically between 6.5 and 6.7 , having 6.6 the usual value [14 16]. Examining Fig. 3 we notice, from this short time ( 2 minutes) experiment, that a slightly lower $\mathrm{pH}$ will cause an increase in the average diameter. We also notice that the small variation of the diameter with the change of the $\mathrm{pH}$ is quite fast, within the first 9 seconds after the $\mathrm{pH}$ change was produced, and we notice this feature from the plot in Fig. 3, where the diameters at $\mathrm{t}=9 \mathrm{~s}$ are already shifted from the initial values, at $\mathrm{t}=0$, which were around $110 \mathrm{~nm}$, considering the errors in determining them using DLS time series processing.

The results of this study are in very good agreement with other studies carried on to investigate the variation of the particles in milk as a result of the change of the $\mathrm{pH}$ and [17] 
is just one of them. A small increase in the milk pH produced an increase in the size of the particles, which proved to be casein micelles [26], and this result is in full agreement with the results reported in this work. In [26] a commercially available zeta sizer was used, therefore the dynamics of the size variation could not be investigated.

The method presented in this work, with the DLS device made from custom parts and the software written to fulfill this purpose, has been used to investigate not only the size change but the dynamic of the size variation, as well. The novelty presented in this work lays in measuring the time required for size changing, and the time was of the order of 7-9 seconds since the change was induced.

\section{Conclusion}

A simplified, but very flexible procedure of DLS that can be used for assessing the average particle diameter of the particles in suspensions has been described in this work. It proved to be robust and totally reproducible, as having a single free parameter during the least squares fit and was used in particle size monitoring. The samples can be prepared directly in the DLS glass tube. The variation of the average bovine milk proteins in aqueous suspensions with the increase in the $\mathrm{pH}$ was investigated during a short time experiment. The experiments revealed that a small increase of the $\mathrm{pH}$ increases the average particles diameter and that this increase occurs in a matter of seconds since inducing the $\mathrm{pH}$ changing.

Acknowledgment: The work presented here was partially supported by the ULBS internal research grants LBUS-IRG-2017-03 and LBUS-IRG-2018-04.

\section{References}

1. J. Vasbinder, Casein - whey protein interactions in heated milk, Ph.D. thesis, ISBN 90-3933194-4, University of Utrecht Press (2002)

2. M. Guo, G. Wang, Polymers 8, 324, (2016), doi:10.3390/polym8090324

3. Y. Piederrière, J. Cariou J,Y. Guern Y, B. Le Jeune, G. Le Brun,J. Lotrian, Opt. Exp. 12, 176 (2004)

4. Y. Piederriere, J. Le Meur, J. Cariou, J.F. Abgrall, M.T. Blouch, Opt. Exp. 12, 4596 (2004)

5. D. Chicea, Eur. Phys. J App. Phys. 40, 305, (2007), doi: 10.1051/epjap:2007163

6. $\quad$ N.A. Clark, J.H. Lunacek, G.B. Benedek, Am. J. Phys., 38 (5), 575 (1970)

7. J.W. Goodman, Laser speckle and related phenomena, Vol.9 in series Topics in Applied Physics, J.C. Dainty, Ed., Springer-Verlag, Berlin, Heidelberg, New York, Tokyo (1984)

8. H.C. Van de Hulst, Light Scattering by Small Particles, New York: Dover Publications (1981)

9. B.J. Berne, R..Pecora, Dynamic Light Scattering: With Applications to Chemistry, Biology, and Physics, Dover Publications (2000)

10. R. Xu, Particle Characterization: Light Scattering Methods, Kluwer Academic Publishers, New York, Boston, Dordrecht, London, Moscow (2002)

11. D. Chicea, Curr. Nanosc. 8(2), 259 (2012)

12. D. Chicea, Meas. Sci. Technol. 28(5) 055206 (2017)

13. D. Chicea, S. Rei, Meas. Sci. Technol. 29105201 (2018)

14. L. Øgendal, Light Scattering Demystified Theory and Practice, University of Copenhagen Press, 2017, retrieved from

http://www.nbi.dk/ ogendal/personal/lho/lightscattering_theory_and_practice.pdf, as of august 2018

15. Einstein, Ann. der Phys. 322 (8): 549 (1905), doi:10.1002/andp.19053220806.

16. D. Dalgleish, A. Law, J. Dairy Res., 55(4), 529, (1988), doi:10.1017/S0022029900033306. 
17. H. Sinaga, N. Bansal, B. Bhandari, Int. J. Food Prop., 20, 179 (2017) DOI: $10.1080 / 10942912.2016 .1152480$ 